\title{
0 PERFIL DE PORTADORES DE DIABETES TIPO 1 CONSIDERANDO SEU HISTÓRICO DE ALEITAMENTO MATERNO
}

\author{
The profile of people with Type 1 Diabetes considering their history of breast feeding \\ El perfil de los portadores de Diabetes tipo 1 considerando su histórico de amamantamiento \\ materno
}

Dalila Teixeira Leal ${ }^{1}$

Flávia Andrade Fialho

Lilian do Nascimento ${ }^{4}$

Wiviam Cristiane Arruda 5

lêda Maria Ávila Vargas Dias ${ }^{3}$

\section{RESUMO}

Trata-se de um estudo que teve como objetivo descrever o perfil dos usuários de um Serviço de Controle de Hipertensão, Diabetes e Obesidade e discutir a relação entre a história de aleitamento materno da criança ou adolescente portador de diabetes tipo 1 e o desenvolvimento desta patologia. A coleta de dados foi realizada por meio de um questionário estruturado, aplicado aos pais ou responsáveis de crianças ou adolescentes portadoras de diabetes mellitus tipo 1, durante o segundo semestre de 2007. 0s dados foram analisados com uma abordagem quantitativa, em que se utilizou a estatística descritiva. Como resultados foram apresentados dados referentes à caracterização da amostra, ocorrência do desmame precoce, período de aleitamento materno exclusivo, dieta oferecida após o desmame, entre outros. Na conclusão é evidenciada a significativa relação entre desmame precoce, com a consequente introdução de substitutos do leite materno e o desenvolvimento do diabetes mellitus tipo 1.

Palavras-chave: Enfermagem. Diabetes Mellitus. Aleitamento Materno.

\begin{abstract}
This study had as objective, describe the profile of the users of the Hypertension, Diabetes and Obesity Control Service and discuss the relationship between the history of breastfeeding of child or teenager who have diabetes type 1 and development of this pathology. The collection of data was realized by a structured questionnaire, applied to parents or responsible for children or teenagers who have diabetes mellitus type 1 , during the second semester of 2007. The data were analyzed in a quantitative approach that was based on a descriptive statistic. As a result it was presented the data referring to sample characterization, occurrence of early weaning, period of exclusive breastfeeding, post-weaning diet, and others. In the conclusion, it is evidenced the importance of a relationship between early weaning, with the consequent introduction of breast milk substitutes, and the development of Diabetes Mellitus Type 1.

Key words: Nursing. Diabetes Mellitus. Breast Feeding .
\end{abstract}

Keywords: Primary Health Care. Tuberculosis. Health Services Accessibility. Therapeutics.

\section{Resumen}

Es un estudio que tuvo como objetivo describir el perfil de los usuarios del Servicio de Control de Hipertensión, Diabetes y Obesidad y discutir la relación entre la historia de amamantamiento materno del niño o del adolescente portador de diabetes tipo 1 y el desenvolvimiento de esta enfermedad. La colecta de los datos fue realizada por medio de un cuestionario estructurado, aplicado en los padres o responsables de estos niños, durante el $2^{\circ}$ semestre de 2007. Los datos fueron analizados en un abordaje cuantitativo en que se utilizó la estadística descriptiva. Como resultados fueron presentados datos referentes a la caracterización de la muestra, ocurrencia de la interrupción del amamantamiento precoz, período de amamantamiento materno exclusivo, dieta ofrecida después de la interrupción del amamantamiento y otros. En la conclusión es presentada la significativa relación entre la interrupción del amamantamiento precoz, con la consecuente introducción de substitutos de la leche materna y el desenvolvimiento de la Diabetes Mellitus tipo 1.

Palabras clave: Enfermería. Diabetes Mellitus. Lactancia Materna.

\footnotetext{
'Enfermeira e pesquisadora Núcleo de Pesquisa em Saúde Materno Infantil e Saúde Coletiva na Faculdade de Enfermagem da Universidade Federal de Juiz de Fora-MG. Brasil. E-mail: lealdalila@hotmail.com, ${ }^{2}$ Acadêmica da Faculdade de Enfermagem da Universidade Federal de Juiz de Fora-MG. Brasil. E-mail: flavinhafialho@bol.com.br, ${ }^{3}$ Professora e Pesquisadora do Núcleo de Pesquisa em Saúde Materno Infantil e Saúde Coletiva da Universidade Federal de Juiz de Fora-MG. Brasil. E-mail: vargasdias@hotmail.com, ${ }^{4}$ Enfermeira e Pesquisadora Núcleo de Pesquisa em Saúde Materno Infantil e Saúde Coletiva na Faculdade de Enfermagem da Universidade Federal de Juiz de Fora-MG. Brasil. E-mail: lilian_sarhdeg@clic21.com.br, ${ }^{5}$ Enfermeira e Pesquisadora Núcleo de Pesquisa em Saúde Materno Infantil e Saúde Coletiva na Faculdade de Enfermagem da Universidade Federal de Juiz de ForaMG. Brasil. E-mail: wivianarruda@bol.com.br
} 


\section{INTRODUÇ̃̃O}

A discussão sobre aleitamento materno vem se evidenciando nos últimos anos em relação à sua importância, duração e fatores que influenciam a decisão de efetivar ou não esse ato. A Organização Mundial da Saúde (OMS) preconiza que a amamentação exclusiva até o sexto mês de vida do bebê, complementada com outros alimentos até os dois anos de vida é o ideal no combate à redução da morbimortalidade infantil, sendo fonte de alimento, de vínculo entre mãe e filho e de proteção contra inúmeras doenças. Além de água, vitaminas e sais minerais, o leite materno contém imunoglobulinas, enzimas, hormônios e anticorpos que protegem o bebê contra infecções. ${ }^{1}$

No reforço das ações em favor da amamentação, são também evidenciados os benefícios à mulher por meio dessa prática, tais como: a redução da hemorragia no pós-parto, por intermédio da estimulação de contrações que levam à involução uterina, processo de fundamental importância, pois a hemorragia pós-parto é considerada uma das principais causas de mortalidade materna no Brasil; redução do risco da mulher contrair câncer de mama; redução do risco de desenvolver anemia, porque não há perda de sangue mensalmente, e com isso o nível de ferro é preservado no organismo; e redução do risco de desenvolver osteoporose na vida madura. ${ }^{2}$

Embora o Governo Federal mantenha o Programa Nacional de Incentivo ao Aleitamento Materno desde a década de 1980, dados do Fundo das Nações Unidas para a Infância (UNICEF) mostram que no Brasil, a taxa de aleitamento materno exclusivo até o sexto mês ainda é muito baixa, em torno de 9,7\%. Para ajudar a reverter essa situação, há hoje no país campanhas de esclarecimento e outras estratégias, como a Iniciativa Hospital Amigo da Criança, que procura envolver os estabelecimentos de saúde em esforços para evitar o desmame precoce. ${ }^{3}$

A revisão bibliográfica é vasta no que diz respeito aos benefícios do aleitamento materno, e, no corolário, os efeitos deletérios do desmame precoce, inclusive apresentase uma possível relação entre o desenvolvimento do diabetes mellitus tipo 1 e a introdução precoce dos substitutos do leite materno ocorrido em decorrência deste desmame. $^{4}$

A etiopatogenia do diabetes mellitus tipo 1 pode estar relacionada com a história de aleitamento materno e dieta oferecida no primeiro ano de vida da criança. A relação se daria devido às propriedades anti-infecciosas do leite materno que deixa de ser oferecido, ou pelo fato de que a amamentação ao seio evitaria que as crianças fossem precocemente expostas a outros agentes etiológicos contidos nos substitutos do leite materno. ${ }^{5}$

0 diabetes mellitus é uma síndrome de etiologia múltipla, decorrente da falta de insulina e/ou a incapacidade da insulina em exercer adequadamente seus efeitos, constituindo uma condição crônica que exige do portador um contínuo autogerenciamento do estilo de vida e adaptação à doença. ${ }^{6}$ A classificação atual do diabetes mellitus inclui três classes principais: diabetes tipo 1, diabetes tipo 2 e diabetes gestacional, sendo que esta classificação é baseada na etiologia, e não no tipo de tratamento. Assim os termos diabetes mellitus insulinodependente e diabetes mellitus não insulinodependente, amplamente utilizados no passado, devem ser substituídos pela terminologia tipo 1 e tipo 2.?

0 diabetes do tipo 1 caracteriza-se pela destruição das células betapancreáticas. Acredita-se que os fatores genéticos, imunológicos e, possivelmente, ambientais contribuam para a destruição dessas células. No diabetes tipo 2, ocorrem principalmente dois problemas, a resistência à insulina e a secreção de insulina comprometida. No diabetes gestacional, ocorre qualquer grau de intolerância à glicose com seu início durante a gravidez. A hiperglicemia desenvolve-se durante a gravidez por causa da secreção de hormônios placentários, o que provoca resistência à insulina. $^{8}$

0 diagnóstico básico da doença se dá através da medida da glicose sanguínea e da presença de sinais e sintomas característicos como: polidipsia, polifagia, poliúria e perda de peso, principalmente quando a hiperglicemia está evidenciada e vem acompanhada da presença de glicose na urina. ${ }^{9}$

Estudos epidemiológicos e experimentais têm mostrado que os fatores ambientais ocupam um lugar de destaque no aparecimento do diabetes mellitus tipo 1, cuja etiologia esteve tradicionalmente vinculada a fatores genéticos, com escassas possibilidades de prevenir o seu surgimento. Esses novos conhecimentos acerca da patogênese do diabetes tipo 1 apontam a possibilidade da prevenção de seu surgimento e a possibilidade de reduzir substancialmente seus efeitos deletérios naqueles que já são portadores dessa doença. ${ }^{5}$

Diante dessa contextualização, definiu-se como objetivo do estudo descrever o perfil dos usuários do Serviço de Controle de Hipertensão, Diabetes e Obesidade (SCHDO) da Secretaria Municipal de Saúde de Juiz de Fora e discutir a relação entre a história de aleitamento materno da criança ou adolescente portador de diabetes tipo 1 e 0 desenvolvimento desta patologia.

Acredita-se que esta discussão possa instrumentalizar o enfermeiro, e demais profissionais de saúde, para a promoção do aleitamento materno, pois à medida que 0 enfermeiro incentiva, acompanha, auxilia e principalmente orienta a tríade mãe-pai-família na efetivação da amamentação exclusiva, contribui para que sejam alcançadas as metas da "Declaração de Innocenti", elaboradas com o objetivo de mobilizar profissionais de saúde e autoridades para mudanças que previnam o desmame precoce. 


\section{MATERIAL E MÉTODO}

Trata-se de um estudo de natureza quantitativa. Este tipo de estudo caracteriza-se pelo emprego da quantificação na coleta e tratamento das informações. Portanto, usa medidas numéricas para testar constructos científicos e hipóteses ou para buscar padrões numéricos relacionados a conceitos cotidianos. ${ }^{10}$

A partir da autorização da Secretaria Municipal de Saúde (SMS) do Município de Juiz de Fora e aprovação do Comitê de Ética em Pesquisa da Universidade Federal de Juiz de Fora, parecer $n^{0}$ 274/2007, foi iniciada uma ambientação com o serviço, o que facilitou a coleta de dados realizada posteriormente, durante o segundo semestre de 2007.

Os sujeitos do estudo foram pais ou responsáveis de crianças ou adolescentes portadoras de diabetes mellitus tipo 1 cadastradas, neste período, no Pólo da Criança e Adolescente Insulinodendente, do SCHDO, que aceitaram participar voluntariamente da pesquisa, respondendo um questionário estruturado aplicado nos dias em que estes compareciam ao serviço acompanhando seus filhos para consulta médica. Constituiu-se uma amostra de 33 mulheres, todas mães de portadores de diabetes mellitus tipo 1 que relataram a história de aleitamento materno de seus filhos.

Após a coleta de dados, foi realizada a sua organização sistemática, constituindo-se na tabulação e categorização, com consequente análise estatística, já que se trata de um estudo de natureza quantitativa. A pesquisa quantitativa considera que tudo pode ser quantificável, o que significa traduzir em números opiniões e informações para classificálas e analisá-las, por meio de recursos e técnicas estatísticas. ${ }^{10}$

Os resultados foram construídos a partir do cálculo de frequência, percentual, média e desviopadrão, constituindo-se em uma estatística descritiva, o que permitiu a elaboração do relatório final, apresentado ao Serviço (SCHDO), aos usuários e à comunidade científica.

\section{RESULTADOS}

$\mathrm{Na}$ caracterização da amostra evidencia-se que, em relação à idade das crianças e/ou adolescentes, filhos das depoentes, houve uma variação de 4 a 18 anos, sendo 11 anos a média da idade; em relação ao sexo, 12 indivíduos $(36,6 \%)$ eram do sexo masculino e $21(63,4 \%)$, do sexo feminino, portanto havendo uma prevalência deste último na amostra considerada. Quanto ao peso ao nascer, foi observada uma variação de $1 \mathrm{~kg}$ a 4,2 kg, com uma média de $2,97 \mathrm{~kg}$. Quanto à idade em que a doença foi diagnosticada, houve uma variação de menos de 1 ano a 16 anos de idade, sendo 8 anos a média. Referente à raça, $26(78,7 \%)$ eram brancos, $3(9,3 \%)$ eram negros, 2 $(6,0 \%)$ eram pardos, e de $2(6,0 \%)$ os pais não souberam definir a etnia.

Tabela 1 - Distribuição da amostra estudada segundo idade, peso ao nascer e idade que desenvolveu o diabetes, Juiz de Fora, 2007.

\begin{tabular}{cccc}
\hline Descrição & Idade (anos) & $\begin{array}{c}\text { Peso ao nascer } \\
\text { (Kg) }\end{array}$ & $\begin{array}{c}\text { Idade em anos em que a } \\
\text { doença foi diagnosticada }\end{array}$ \\
\hline Média & 11,55 & 2,97 & 8,29 \\
D.P & 5,06 & 0,79 & 4,76 \\
Mínimo & 4 & 1,00 & 1 \\
Máximo & 18 & 4,20 & 16 \\
\hline
\end{tabular}

0 desmame precoce foi constatado em 29 indivíduos, correspondendo a $87,9 \%$ da população estudada. É oportuno destacar que, no presente estudo, desmame precoce foi definido de acordo com as orientações do Ministério da Saúde como aquele ocorrido antes do sexto mês de vida. ${ }^{1}$

A Tabela 2 mostra o período de aleitamento materno exclusivo e evidencia que entre os entrevistados 10 das crianças $(30,6)$ foram aleitadas exclusivamente ao peito por menos de 1 mês, 2 crianças
$(6,0 \%)$ tiveram aleitamento materno exclusivo por até 1 mês, 4 crianças $(12,1 \%)$ receberam leite materno exclusivo por até 2 meses, $5(15,1 \%)$ foram amamentadas exclusivamente por até 3 meses, nenhuma das mães relatou ter amamentado exclusivamente por 4 ou 5 meses, 6 das crianças $(18,2 \%)$ receberam leite materno exclusivo por até 6 meses e $3(9,0 \%)$ não receberam leite materno, sendo que 3 dos participantes $(9,0 \%)$ não souberam precisar o tempo de aleitamento exclusivo. 
Tabela 2 - Distribuição da amostra estudada segundo o período de aleitamento materno exclusivo, Juiz de Fora, 2007.

\begin{tabular}{ccc}
\hline Período & Frequência & Percentual (\%) \\
\hline Não recebeu leite materno & 3 & 9,0 \\
Menos de 1 mês & 10 & 30,6 \\
Até 1 mês & 2 & 6,0 \\
Até 2 meses & 4 & 12,1 \\
Até 3 meses & 5 & 15,2 \\
Até 4 meses & 3 & 9,0 \\
Até 5 meses & 2 & 6,0 \\
A té 6 meses & 4 & 12,1 \\
Total & $\mathbf{3 3}$ & $\mathbf{1 0 0 \%}$ \\
\hline
\end{tabular}

Evidencia-se que, das 29 depoentes que relataram ter desmamado seu filho antes dos seis meses, $10(34,4 \%)$ promoveram o desmame precoce por vontade própria; $4(13,8 \%)$ relataram o cessar da produção láctea como motivo do desmame; 6 $(20,7 \%)$ atribuíram a condições clínicas maternas; $4(13,8 \%)$, a condições clínicas da criança e 5 $(17,3 \%)$, ao fato de possuírem leite fraco, o que se sabe ser um mito.
A Tabela 3 mostra a variedade de alimentos oferecidos as crianças após o desmame, sendo o leite de vaca, na forma integral ou associado, o alimento oferecido com maior frequência após o desmame, correspondendo a $52 \%$, ou seja, utilizado por 15 crianças, seguido do leite industrializado que foi oferecido a 13 crianças (38\%). Vale ressaltar que participaram desta análise somente as crianças que foram desmamadas antes dos 6 meses, portanto, um total de 29 crianças, correspondendo a $87,9 \%$ da amostra.

Tabela 3- Distribuição da amostra estudada segundo dieta oferecida após o desmame precoce. Juiz de Fora, 2007

\begin{tabular}{ccc}
\hline Alimentos & Frequência & Percentual (\%) \\
\hline Leite de vaca integral & 4 & 13,8 \\
$\begin{array}{c}\text { Mingau de amido de milho } \\
\text { com leite de vaca } \\
\text { Leite industrializado }\end{array}$ & 9 & 31 \\
Leite de vaca diluído & 13 & 44,8 \\
Leite de cabra & - & - \\
Mingau de arroz com leite \\
de vaca \\
Outros & - & - \\
& 2 & 3,9 \\
\hline
\end{tabular}

Referente ao histórico familiar de diabetes observou-se que enquanto 18 pessoas $(54,4)$ negaram, 15 pessoas $(45,6 \%)$ confirmaram, não sabendo especificar o tipo de diabetes. Destas, 11 pessoas $(72 \%)$ responderam que os avós eram os parentes acometidos; $2(14 \%)$, o pai e 2 (14\%), a mãe.

Questionadas quanto ao aparecimento de algum problema de saúde em decorrência do diabetes, 26 pessoas (76\%) negaram e 8 pessoas (24\%) afirmaram o aparecimento de alguma situação patológica, tais como: fibromialgia e esofagite; catarata, glaucoma e outros problemas visuais; problemas renais; amigdalites recorrentes; deficiência na cicatrização; infecções urinárias e herpes-zóster. 


\section{DISCUSSÃO}

Os resultados do estudo permitiram evidenciar a alta prevalência do desmame precoce no histórico das crianças e adolescentes, hoje portadoras de diabetes mellitus tipo 1 , destacando a importância do aleitamento materno exclusivo, considerado um fator de proteção ao desenvolvimento de patologias.

0 desmame precoce é resultado de uma complexa interação de fatores socioculturais, como, por exemplo, o processo de industrialização, o surgimento e a divulgação de leite industrializado, a adesão de profissionais de saúde a prescrição de alimentação artificial, a inserção da mulher no trabalho e a adoção nas maternidades de medidas pouco incentivadoras do aleitamento materno, medidas que acarretam prejuízos físicos, emocionais, econômicos e sociais para a criança e sua família, uma vez que dificultam a prática do amamentar. ${ }^{11}$

Uma estratégia importante de incentivo ao aleitamento materno é a instituição de bancos de leite. Algumas mulheres, quando estão amamentando, produzem um volume de leite além da necessidade do bebê, o que possibilita que sejam doadoras de um Banco de Leite Humano. De acordo com a legislação que regulamenta o funcionamento dos Bancos de Leite no Brasil (RDC No 171), a doadora, além de apresentar secreção láctea superior às exigências de seu filho, deve ser saudável e se dispor a ordenhar e a doar o excedente.

Quanto ao período de aleitamento materno exclusivo, com base nas respostas, evidencia-se que a maior parte das crianças foram aleitadas exclusivamente ao peito por menos de 1 mês, no que sobressai a importância do primeiro mês de vida no sucesso do aleitamento materno; portanto, os profissionais de saúde devem criar estratégias que contribuam para a efetivação do mesmo neste período, já que o preconizado é que a amamentação seja exclusiva até os 6 meses e complementada com outros tipos de alimento até os 2 anos de vida ou mais.

Referente à dieta oferecida após o desmame, o alimento mais utilizado foi o leite de vaca, na forma integral ou associado ao amido de milho ou de arroz, seguido do leite industrializado. 0 que é um dado preocupante, pois estudos apontam o leite materno e o leite de vaca, respectivamente, como fator de proteção e de risco para o diabetes mellitus, tipo $1 .{ }^{12}$

A exposição precoce ao leite de vaca pode ser um importante determinante do diabetes mellitus tipo 1 , considerando a constatação de que $30 \%$ dos casos de diabetes mellitus tipo 1 poderiam ser evitados se as crianças até 3 meses não recebessem leite de vaca, já que existe a relação entre a introdução precoce da alimentação complementar com o desenvolvimento de doenças atópicas. Essa relação foi pautada nas evidências sorológicas que mostram altas concentrações de anticorpos antialbumina bovina entre os diabéticos do tipo $1 .{ }^{13}$

Foi demonstrado em um estudo a alta prevalência de anticorpos antialbumina bovina sérica em pacientes portadores de diabetes mellitustipo 1 de início recente (100\%) em relação ao grupo controles de não diabéticos (3,8\%). A proteína sérica bovina contém uma região de 17 aminoácidos diferente de qualquer sequência de aminoácidos de albumina sérica humana, mas que se assemelha a uma outra proteína encontrada na superfície da célula betapancreática. Os anticorpos contra este epítopo reagem também de maneira cruzada com a referida proteína humana da superfície da célula beta e podem ser os responsáveis pelo desencadeamento do processo autoimune que leva ao diabetes mellitus tipo $1 .{ }^{4}$

A albumina bovina sérica contida no leite é introduzida precocemente no recém-nascido ainda com uma barreira gástrica imatura. Esse peptídeo poderia induzir uma reação imune contra essa proteína, levando a um quadro de diabetes mellitus tipo 1, que tem como mecanismo patogênico fundamental a destruição autoimunológica das células betapancreáticas, que se assemelham à estrutura da albumina bovina sérica. ${ }^{7}$

Nos resultados obtidos referentes aos motivos do desmame, observa-se que a vontade da mãe foi predominante no processo de desmamar. Sabe-se que fatores culturais socialmente construídos ao longo da existência constituem barreiras para o prolongamento do aleitamento materno exclusivo, o que gera a necessidade do acesso à informação e a desmistificação, para que novos construtos sejam alicerçados na sociedade.

Um dado apresentado nos resultados que merece ser esclarecido é o fato de as participantes terem atribuído o desmame à produção de leite fraco. Ressalta-se que isso é um mito, pois fisiologicamente essa possibilidade não existe. Esse dado vai ao encontro do estudo que, ao avaliar a prevalência do aleitamento materno exclusivo nos primeiros três meses de vida e os fatores intervenientes, apontou como causa do desmame e da amamentação não exclusiva fatores relacionados à mãe, como a errônea crença de que o leite materno não é suficiente em volume ou qualidade para suprir as necessidades do lactente. ${ }^{14}$

Os resultados mostram que a maioria das crianças não possui histórico familiar de diabetes; entretanto, para um expressivo número de participantes, esse histórico foi evidenciado. Em um estudo epidemiológico, ao analisar a ocorrência de casos de diabetes na família, foi constatado que $41 \%$ dos pacientes apresentavam parentes com diabetes mellitus; desses, 5 (8,5\%) eram pais, 29 (49,2\%) eram avós e 25 (42,3\%), tios ou primos. ${ }^{15}$ Tal relação foi evidenciada em nosso estudo, o qual mostrou uma predominância dos avós como parente acometido pelo diabetes no histórico familiar.

Referente aos problemas de saúde em decorrência do diabetes mellitus tipo 1, constatou-se que a maioria referiu não apresentar até o momento nenhum comprometimento. Entretanto sabe-se que o problema principal do diabetes mellitus tipo 1 são seus efeitos ao longo do tempo. 
Como resultado das alterações glicêmicas, é possível que o portador desenvolva complicações crônicas no decorrer da vida, como distúrbios cardiovasculares, renais, neurológicos e visuais. Existem também complicações agudas que ocorrem a partir de crises de hipoglicemia ou de hiperglicemia, sendo esses quadros mais comuns na adolescência, porque os jovens tendem a exagerar no consumo de açúcares ou na prática de exercícios, bem como negligenciar os horários de aplicação da insulina, o que interfere diretamente na homeostase, gerando o quadro de descompensação diabética. ${ }^{16}$

Considerando que o surgimento de agravos à saúde ocorre com o passar dos anos, e sendo a população foco deste estudo crianças e adolescentes, identifica-se 0 acompanhamento desses sujeitos como um dado impor tante para ser investigado em pesquisas prospectivas. Além da importância da atuação do enfermeiro, que desenvolve papel primordial na prevenção, terapêutica e recuperação dos pacientes portadores de diabetes, já que o foco do cuidado de enfermagem para esses pacientes é desenvolver uma sólida base educacional, visando ao domínio de conceitos e habilidades necessárias ao tratamento a longo prazo da patologia e de suas complicações.

\section{CONCLUSÃO}

Este foi um estudo quantitativo, realizado a fim de traçar o perfil dos usuários do SCHDO e relacionar o desenvolvimento do diabetes com a história pregressa de seu aleitamento. Além do alcance dos objetivos propostos inicialmente, novos objetivos surgiram no decorrer das entrevistas, balizando para a realização de outras pesquisas, em especial, pesquisas com enfoque qualitativo, que permitam a expressão de sentimentos em relação à doença.

Ao findar a pesquisa, fica deflagrada a importância da discussão sobre aleitamento materno no que diz respeito ao seu cumprimento e fatores de influência na sua decisão e duração, bem como a necessidade de se trabalhar mais a promoção do aleitamento natural, em livre demanda, até os seis primeiros meses de vida da criança, pois se acredita que, com a implementação deste ato, seja minimizada a introdução precoce de outros alimentos, os quais podem estar associados ao aparecimento do diabetes mellitus tipo 1 e outras doenças.

Nesse sentido, o enfermeiro tem um impor tante papel na promoção do aleitamento, podendo incentivar esse ato através de campanhas, educação direta com a população, trabalhos em grupos educativos e, principalmente, ajudando as mães no desenvolvimento da prática do aleitar, considerando que essa é uma questão que envolve toda a família.

As medidas utilizadas pela Organização Mundial da Saúde e o Fundo das Nações Unidas para promover essa prática visam à redução da morbimortalidade infantil, devido ao fato de o leite materno promover laços de afeto entre mãe e filho e proteger a criança contra inúmeras doenças. Entretanto, mesmo com o Programa Nacional de Incentivo ao Aleitamento Materno, criado desde a década de 1980, a literatura mostra que a taxa de aleitamento materno exclusivo até o sexto mês ainda é muito baixa no País, devendo os profissionais de saúde ter como meta transformar essa realidade. Os dados dessa pesquisa vêm corroborar essa afirmativa, visto a expressiva ocorrência do desmame precoce. Assim, vale ressaltar que é significativa a relação entre o desenvolvimento do diabetes mellitus tipo 1 e o desmame precoce, com sua consequente introdução de substitutos do leite materno. Porém, entende-se que não foi possível esgotar esse dado, demandando, assim, a realização de mais pesquisas com essa temática, especialmente pesquisas que utilizem grupo-controle.

\section{REFERÊNCIAS}

1- Organização Mundial da Saúde (OMS). Proteção, promoção e apoio ao aleitamento materno: o papel especial dos serviços materno-infantis. Genebra: OMS; 2003.

2- Oliveira PMP, Rebouças CBA, Pagliuca LMF. Literatura de Cordel como meio de promoção para o aleitamento materno. Esc Anna Nery. 2008 jun; 12 (2): 217 - 23.

3- Fundo das Nações Unidas para a Infância- UNICEF. Infância ameaçada: situação mundial da infância. Nova York(USA); 2004.

4- Macedo CLD, Ferreira MC, Naujorks AA, Tercziany A, Costa FJB, David HC, et al. Aleitamento materno e diabetes mellitus do tipo 1. Arq Bras Endocrinol Metabol. 1999 out; 43 (5): 360-65.

5- Medeiros IS, Rivera MAA, Benigna MJC, Cardoso MAA, Costa MJC. Estudo de caso-controle sobre exposição precoce ao leite de vaca e ocorrência de diabetes mellitus tipo 1 em Campina Grande, Paraíba. Rev Bras Saude Materno Infant. 2003 jul; 3(3): 271-80.

6- Santos Filho CV, Rodrigues WHC, Santos RB. Papéis de autocuidado: subsídios para enfermagem diante das reações emocionais dos portadores de diabetes mellitus. Esc Anna Nery. 2008 nær,12(1): $125-29$.

7- Coronho V, Petroianu A, Santana EM, Pimenta LG. Tratado de endocrinologia e cirurgia endócrina. Rio de Janeiro (RJ): Guanabara Koogan; 2001.

8- Brunner LS, Suddarth DS. Tratado de enfermagem médico-cirúrgico. $10^{\mathrm{a}}$ ed. Rio de Janeiro (RJ): Guanabara Koogan; 2006.

9-Ministério da Saúde (BR). Diabetes mellitus. Cadernos de Atenção Básica, 16. Brasília (DF); 2006.

10- Thomas JR, Nelson JK. Métodos de pesquisa em atividade física. $3^{\text {a }}$ ed. Porto Alegre (RS): Artmed; 2002.

11- Araujo MFM. Situação e perspectivas do aleitamento materno no Brasil. In: Carvalho MR, Tamez RN. Amamentação: bases científicas para a prática profissional. Rio de Janeiro (RJ): Guanabara Koogan; 2002. p 1-10. 
12- Gimeno SGA, Souza JMP. Amamentação ao seio, amamentação com leite de vaca e o diabetes mellitus tipo 1: examinando as evidências. Rev Bras Epidemiol. 1998; 1(1): p 4-13.

13- Monte CMG, Giugliani ERJ. Recomendações para alimentação complementar da criança em aleitamento materno. J Pediatr, Rio de Janeiro, 2004 nov; 80(5): 131-41.

14- Neto ETS, Oliveira AE, Zandonade E. 0 aleitamento materno exclusivo nos primeiros três meses de vida. São Paulo (SP): Cortez; 2007.

15- Josino MV. Diabetes mellitus tipo 1: estudo epidemiológico e $\mathrm{m}$ um centro de referência no sul de Brasil. [tese]. Florianópolis (SC): Faculdade de Medicina, Universidade Federal de Santa Catarina; 2004.

16-Ballas YG, Alves ICB, Duarte WF. Ansiedade em adolescentes portadores de Diabetes mellitus. Bol Psicol. 2006 jan; 56 (124): 111-25. 